\title{
Follower behavior under stress in immersive VR
}

\author{
Alejandro Ríos · Nuria Pelechano
}

Received: date / Accepted: date

\begin{abstract}
Understanding human decision making is a key requirement to improve crowd simulation models so that they can better mimic real human behaviour. It is often difficult to study human decision making during dangerous situations because of the complexity of the scenarios and situations to be simulated. Immersive virtual reality offers the possibility to carry out such experiments without exposing participants to real danger. In the real world it has often been observed that people tend to follow others in certain situations (e.g: unfamiliar environments or stressful situations). In this paper we study human following behaviour when it comes to exit choice during an evacuation of a train station. We have carried out immersive VR experiments under different levels of stress (alarm only or alarm plus fire) and we have observed how humans consistently tend to follow the crowd regardless of the levels of stress. Our results show that decision making is strongly influenced by the behavior of the virtual crowd: the more virtual people running the more likely are participants to simply follow others. The results of this work could improve behavior simulation models during crowd evacuation, and thus build more plausible scenarios for training firefighters.
\end{abstract}

Keywords crowd following · immersive VR $\cdot$ studies of human behavior

\author{
A. Ríos \\ Universitat Politècnica de Catalunya \\ Tel.: +123-45-678910 \\ E-mail: arios@cs.upc.edu \\ N. Pelechano \\ Universitat Politècnica de Catalunya \\ Tel.: +134-93-417858 \\ E-mail: npelechano@cs.upc.edu
}

This is a post-peer-review, pre-copyedit version of an article published in Virtual reality. The final authenticated version is available online at: http://dx.doi.org/10.1007/s10055-020-00428-8 


\section{Introduction}

Crowd simulation models can have a big impact on the overall believability of a populated environment. Given the variety of applications, such as video games, movies or training, it is important to tailor the behavior of the crowd to the particular simulation. In order to simulate realistically different types of crowds and behaviors it is essential to first study how humans behave in such scenarios.

Complex simulations such as emergencies, fire situations or evacuations are expensive and difficult to implement in the real world, thus virtual environments can be used instead. VE are often populated with a crowd to increase the level of presence [29] and this leads to participants behaving as they would do in the real world. This fact has led many research groups to study human behavior in virtual reality environments [24].

Most of the work dealing with immersive crowds has focused on studying low level reactive behavior (e.g. collision avoidance), however there has been little work studying higher level decision making in such environments.

In order to develop more accurate pedestrian evacuation models, it is necessary to study human behavior under these circumstances, and to find the relationships between individuals' personalities and their behavior. By doing so, we could achieve human models that more realistically simulate the decision making, route selection, or the inclination to follow others' movement. Most crowd evacuation models, assume that individuals know their way around, and will automatically run towards the closest exit. In this cases, egress depends exclusively on the formation of bottlenecks, the flow rates through doors, or the densities that appear in the environment. However, in a real scenario, there would be a large variety of personalities with different levels of knowledge about the environment that will influence their decision making.

Our main goal with this paper is to study to what extend people follow others during an evacuation. Given an everyday situation such as walking in a train station, suddenly a train gets on fire, an alarm goes off and people starts to evacuate the station. We want to study human decision making when looking for an exit depending on the crowd behavior under low stress levels. We achieve this by having an alarm going off, and test whether the presence or absence of fire has an impact on decision making and nervousness. The findings of this work could be used to develop crowd simulation models that more accurately simulate human decision making. Such behavior models could allow us to build more plausible scenarios for training firefighters and police in case of emergencies.

\section{Related Work}

In this section we survey previous works related to crowd simulation, collision avoidance in immersive virtual environments, gaze and interaction, immersion and stress in VR and decision making models. 


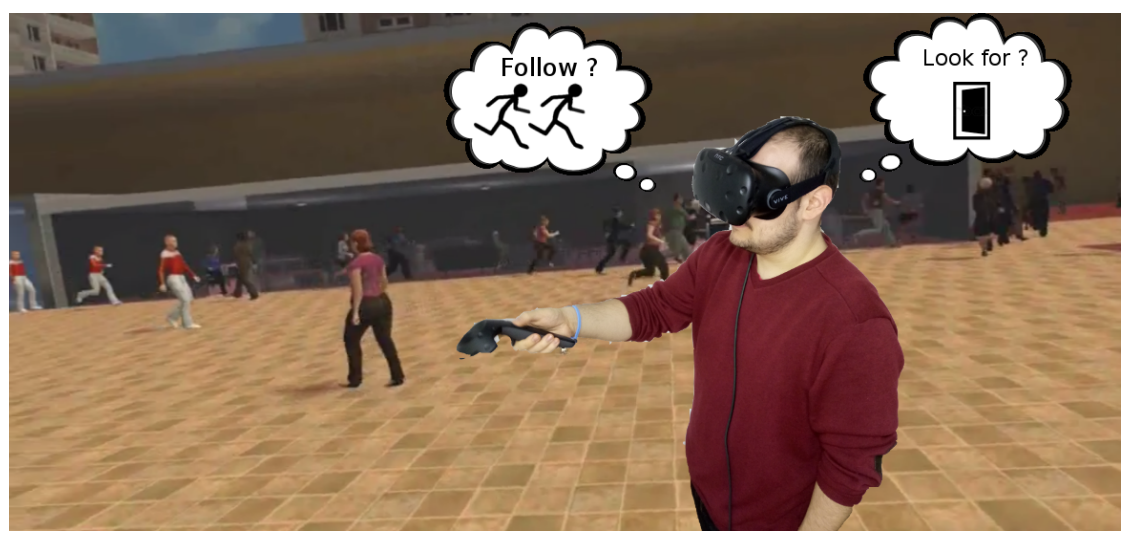

Fig. 1: Participant testing the evacuation scenario. As an alarm goes off, the participant will have to decide between looking for an exit, or following others.

\subsection{Crowd simulation}

Crowd simulation is a research field where validation can become an extremely challenging problem. Many approaches have been presented to tackle such a difficult problem. Quantitative measurement of features (densities, speeds, formations, etc. $[4,14,15,19,39])$ offer an estimate of how close the simulated crowd is to data gathered from real humans, however it may give overall good results and yet the simulation not feel natural to the human eye.

Perception studies have been carried out to evaluate crowd behavior from a human perspective $[7,8]$, however, in the real world we rarely observe crowds from an outsider point of view. Therefore there is a need to perform such perceptual evaluations from a first person point of view. Immersive Virtual Environments offer a platform to study human behavior as long as the participant experiences high levels of presence which will lead to real responses to the virtual characters [30], [27], [20].

There are crowd simulation models that incorporate personality and psychological state to affect human movement, but it is typically limited to modifying speeds, queuing behavior, or personal space $[13,17,28]$. It is thus necessary to better understand how elements such as stress or nervousness can affect human decision making to be able to closely simulate it.

\subsection{Collision Avoidance}

The first steps towards using Immersive Virtual Environments as a validation tool have focused on studying the impact of different collision avoidance algorithms on the level of presence experienced by the user [29], [34], [1]. Gupta et al. evaluated the impact of manipulating the instructions given to the participants to increase the engagement with a virtual crowd [11]. Bruneau et al. 
studied collision avoidance strategies against group of agents based on their appearance and formation [3]. [25] and [31] also studied collision avoidance but only between two persons walking along crossing trajectories. Turner and Penn proposed a model to simulate agents' movement based on visibility which has been evaluated against real data of pedestrians' space occupancy; agents move towards the most available space within their field of view [36] or in the direction where they could see further and thus would open more possibilities for exploration [37]. Visibility approaches have also been used to simulate collision avoidance, by implementing a synthetic vision algorithm to compute time to collision and determine the most immediate hazards to avoid [26]. Immersive Virtual Environments have been used to develop platforms to gather data on human locomotion around virtual obstacles [2], and collision avoidance manoeuvres when walking through a virtual crowd [24]. The work by Rojas et al. focused on simulating group behavior and then used immersive VR with a head mounted display to evaluate the model when the participant was included in the group [32].

\subsection{Gaze and interaction}

How virtual agents act in the VE and body ownership have an impact on participants reactions when immersed in the VE as shown in [23], [10] and [35]. In [35], participants are immersed in a dramatic situation in which a group of refugees wait for a boat on a shore in Turkey to be taken to Europe. The design of the environment was modulated by two factors: responsiveness and embodiment. Responsiveness is associated to the fact that the virtual agents respond or look at the participant when she looked at them. Embodiment referred to whether the participant would see a virtual body when she looked down. Their results proved that both factors contributed positively to the sense of presence and plausability. In [23] participants preferred a system in which virtual agents move in a natural way while gazing at the user in the virtual environment.

\subsection{Immersion and stress in VR}

Virtual Reality has also been used to simulate a stressful scenario. The decreasing price of Virtual Reality hardware allows researchers and therapists to develop applications that help patients to reduce their levels of stress and anxiety related to phobias or other disorders ( [21]). Post-traumatic stress disorder, flying or spider phobias can be treated in a virtual reality environment resulting in a reduction of physiological symptomatology similar to in vivo exposure. A few sessions of Virtual Reality Environment treatment for some phobias lead to significant improvements in anxiety levels. VRE are much cheaper and can be modified and controlled in an easier way than real environments such as take-off situations to treat flying phobia. A good review of 
virtual reality applications related to psychology, sociology and neuroscience can be found in [9].

[22] presented an experiment in which 36 real participants performed several tests in a $3 \mathrm{D}$ environment under a low and a high stress situation. One of the tests consisted of escaping from an environment with 4 possible exit locations. Only one of these exits was functional whereas the rest were blocked. Just a few of the participants knew about the safe exit and the rest were informed about this fact. Stress was implemented using time pressure, a reward system and environmental factors such as red blinking lights and fires. All participants performed each test at the same time in a big laboratory and though they were not immersed in a crowded virtual environment, they showed similar behaviors as in a real scenario. Their results showed that pedestrians have a higher probability to follow others when the density of neighbouring is higher.

\subsection{Decision making}

There are a lot of studies in the social psychology literature about crowd behavior. For example, the contagion effect appears as the propensity for individuals in a crowd to unquestioningly follow the predominant ideas and emotions of the crowd [16]. It appears that the anonymity of the crowd makes the individuals within it, lose their sense of responsibility and individual self. It is thus interesting to study whether virtual crowds have also a similar influence on individuals' behavior. According to Cialdini, there is a heuristic most of us use to determine what to do, think, say, and buy, which is The principle of social proof. This principle says that we look at what other people are doing in order to learn what is correct [5]. According to Coultas [6], when an individual joins a group, copying the behaviour of the majority would be a sensible, adaptive behaviour, which facilitates acceptance into the group and can lead to survival when it involves taking decisions. According to her studies, modern humans have inherited the adaptive behaviors of our ancestors, for whom awareness of others helped them survive in a dangerous and uncertain world.

There have been many approaches to simulate high level decision making for autonomous agents and crowds. For example the work by Van Toll et al. assumed agents decide the paths to follow by combining information regarding path length and density [38]. Guy et al. computed paths based on the idea that humans try to minimize the effort to reach a destination [12]. These methods compute paths assuming that humans tend to somehow optimize their trajectories, however more studies are needed to determine how humans decide paths in the real world. If we think of a large city, chances are that people choose paths based on width of the streets, how busy they are, or whether there are shops of interest.

There has been work on crowd simulation during an evacuation based on the 'follow the leader' behavior [28]. Quantitative measurements during an evacuation were taken and compared based on the percentage of people exhibiting a follower behavior as opposed to those exploring on their own 


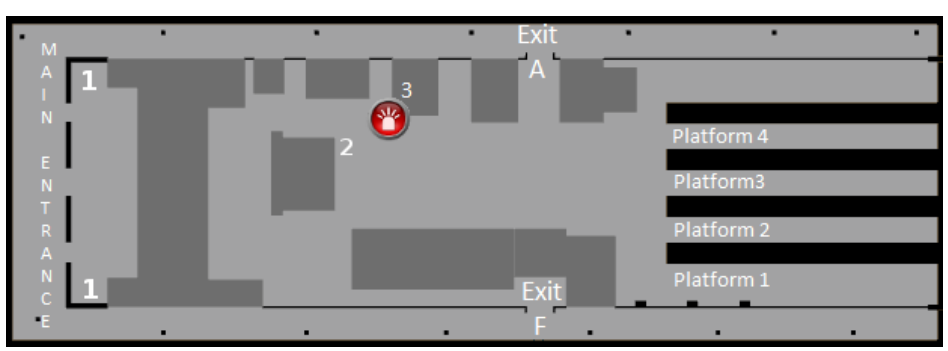

Fig. 2: Layout of the virtual train station, showing the location of the tickets machines (labeled with number 1), the drinks machines (labeled 2), the platforms, the green drinks machine (labeled 3), the exits and the position where the participant will be when the alarm goes off (red icon next to 3).

looking for an exit. The results showed how having a certain combination of followers and experts, would lead to the best evacuation times, however it was unclear which would be the real percentage of 'followers' observed in a real situation. Obtaining such data from a real scenario is hard, and thus in this work we want to study what is the likelihood of humans following others during an emergency situation.

Virtual reality has been extensively used to simulate environments and study human reactions. These experiments try to reproduce a real situation in a virtual environment, reducing both the expense and the risk involved in real life. [10] and [18] are good examples on how an accurate virtual reality simulation can help to investigate psychological phenomena, decision making and human behaviour.

The work presented in this paper is an extension of [33] in which preliminary results related to follower behavior were presented without a thorough statistical analysis. In this paper the virtual environment in [33] has been expanded with the presence of a fire and further studies have been carried out to study decision making and level of nervousness.

\section{Experiment}

\subsection{Design}

The goal of our experiment is to induce users to navigate a virtual train station while interacting with different elements. The train station is populated with a virtual crowd exhibiting an everyday behavior (e.g. buying tickets, going to the platforms and getting on and off the trains, walking in the shops, etc.). The layout of our virtual train station is inspired by the Valencia Nord train station, Figure 2 shows the map of the station indicating location of elements, exits and participant position when an alarm is set off. 


\subsection{Physical Set-up}

All the experiments were conducted in a $1.5 \times 2 \mathrm{~m}$ indoor lab area since it is not necessary for the participants to move physically. Position tracking was performed using an HTC VIVE immersive virtual display and a VIVE controller was used to move around the virtual space to carry out the different tasks. Touching or pressing the front area of the VIVE controller (marked as a green dot in Fig. 3) the participant can move through the environment walking or running. The direction of movement was given by the pointing direction of the controller. The surroundings can be inspected by moving the head. In the station, over a hundred avatars move around simulating an everyday situation. The participants also wore earphones to hear the typical sounds in a train station (the train announcements, people talking, steps, etc.). Unity Game Engine was used to render the environment and animate the avatars.
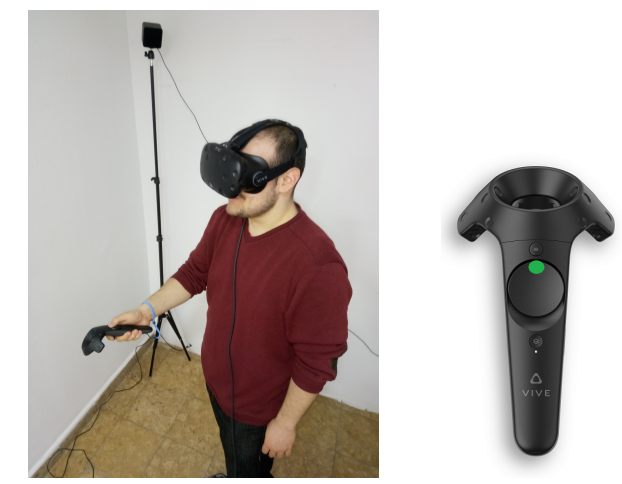

Fig. 3: On the left, participant wearing the HTC VIVE head mounted display, while interacting with the VE using the controller. On the right, controller with the green point showing where the user has to touch for walking or press for running.

\subsection{Procedure}

Before executing the experiment, participants read a document with information and instructions about the simulation such as how to use the controller to interact with the virtual objects or navigate through the environment. They also signed a consent form with information regarding the possibility of dizziness while performing navigation using an HMD, and were told that they could leave the experiment at any time if needed.

When the simulation starts the user is located on the street in front of the main entrance of the train station. In addition to the main entrance, the train station has two more exits: one located at the center of the shopping 
area (named exit A) and another one located behind the shops, in one of the side aisles and less accessible from the main area (named exit F). Both exits can be seen in (see Fig. 2).

Tasks are shown to the participant in a semitransparent text box, that appears partly overlapping the virtual environment for a few seconds, at the user eye level. Once a task is performed, the next task is shown (see Fig. 4).

The tasks are sorted in a specific way to make the participant walk through the entire station, starting from the main entrance and arriving to the platforms that are located at the far end. Tasks involve the participant looking for a vending machine (tickets or drinks) or to be placed on a specific platform. To complete a task related to a vending machine the user must approach the VIVE controller to the machine until a collision with a trigger is detected and a blink occurs. After that, the next task appears for a few seconds.

Figure 5 shows a sequence of screen-shots from a simulation with some of the tasks described above.

Two groups of participants performed the experiment in the same scenario but under two different stress conditions (alarm only, or alarm and fire). For the first group, the list of tasks performed was

- Task 1: Look for a tickets machine.

- Task 2: Look for a drinks machine.

- Task 3: Go to Platform 1.

- Task 4: Look for a green drinks machine.

- Task 5: Find an Exit

Once the task "Look for a green drinks machine" is performed, an alarm goes off and a red emergency light flashes continuously which is visible from the entire station, and it is accompanied by an alarm noise (see Fig. 7).

For the second group, there was an additional task, that was added to guarantee that all participants would see the fire as the alarm would go off. This additional task was "Go to platform 3" and it appeared after Task 4 was performed. This new message would make the user face the platforms and as soon as the participant would move towards platform 3, the alarm and fire would go off and the final task 'Find an Exit' would be shown. The situation of

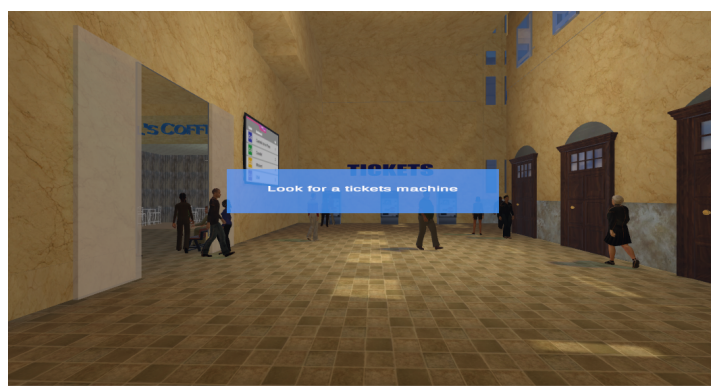

Fig. 4: Example of how the next task to perform is shown to the user. 


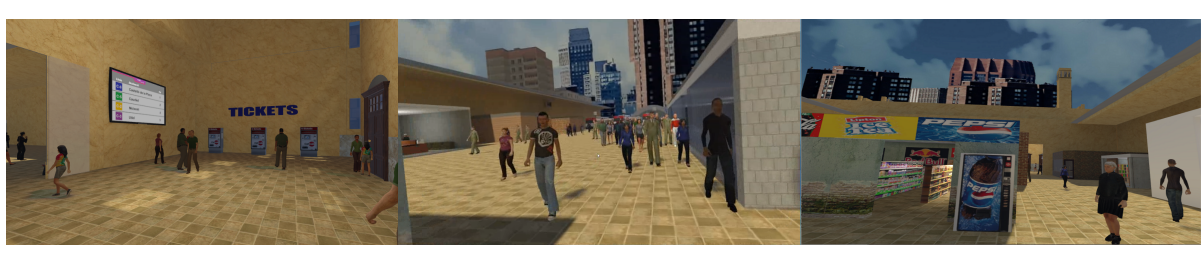

(a)

(b)

(c)

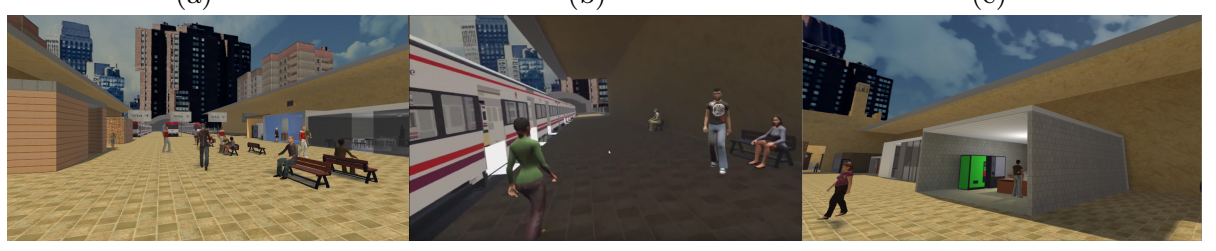

(d)

(e)

(f)

Fig. 5: Sequence of images showing the train station and the tasks to be performed: (a) Tickets machines located at the entrance hall (task 1). The screen shows the next train departures (platform and time). Audio announcements are also given through speakers. (b) Crowded shopping area near the entrance. (c) A drinks machine (task 2). (d) Walking towards the trains. (e) Platform number 1 (task 3). (f) The green drinks machine (task 4).

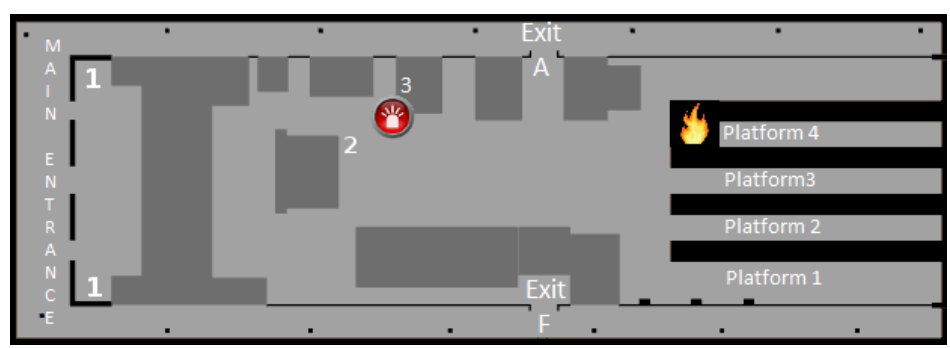

Fig. 6: The position of the fire after the participant performs task 4 .

the fire can be seen in Fig. 6. Note that this task hardly affects their trajectory, as it appears far away from the possible exit routes (see Fig. 8).

In both situations participants are informed that they must find an exit, while they can observe that a certain number of virtual agents begin to run in the direction of the least obvious exit (Exit F, which is the one hidden in the alley behind some shops). Meanwhile, the doors of the main entrance get locked when the alarm goes off, so participants would have to look for an alternative exit in the eventual case that they tried to exit through them. The simulation ends when participants find an exit. 


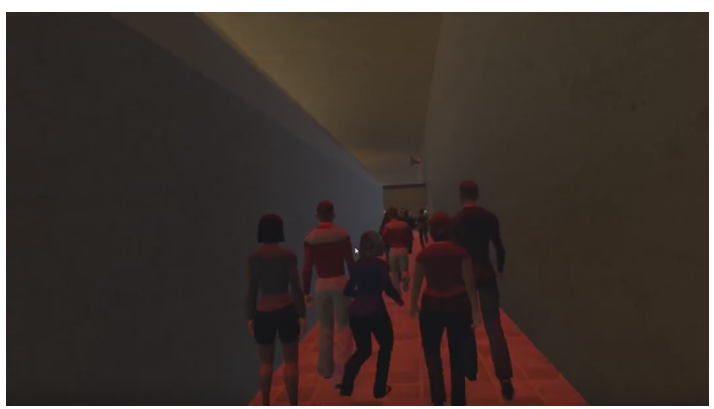

Fig. 7: Alarm going off.

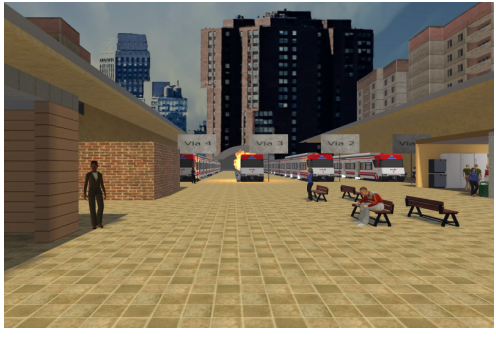

(a)

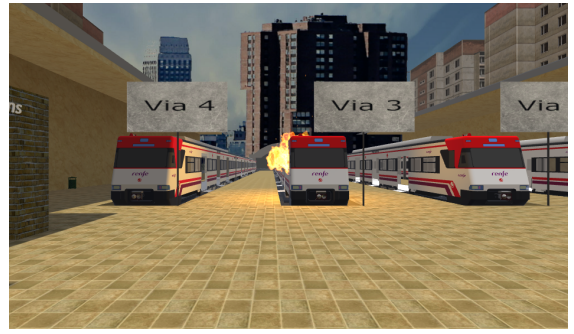

(b)

Fig. 8: Images showing the fire in one of the trains: (a) The fire seen from the user position after performing task 4. (b) A closer view.

\subsection{Participants}

A total of 47 subjects participated in the experiment (34 males and 13 females) aged from 18 to 70 who had not been previously warned about the emergency situation that they would experience during the simulation. 43 of them had a lot of experience with computers, 13 had good experience with virtual reality and 30 had played a lot of video games before the experiment. The participants were divided into two groups in a between-group design: in the first group 22 participants performed the experiment with the fire off, so they could only hear the alarm and see the flashing light; in the second group 25 participants could see the fire on the way to the platforms while hearing the alarm and seeing the flashing light. Each participant could perform the experiment only once since knowing when and where the alarm and the fire are would affect their behaviour in the simulation.

\subsection{Scenarios}

Since the purpose of this experiment was to study the impact that crowd behavior had in user's decisions, the experiments were carried out under three 
different conditions for both the two groups. The independent variable was the percentage of avatars that would start running towards the most hidden exit (Exit F) as soon as the alarm would go off. We tested with $0 \%$ as the base case, $50 \%$ and $100 \%$ of the avatars exhibiting the follower behavior. Each participant experienced only one of the conditions. The virtual characters that did not run towards the exit kept wandering around the train station carrying out randomly generated goals and animations, as if nothing was happening. 18 participants tried the scenario with $100 \%$ of virtual agents behaving as followers and running towards Exit F (9 without fire, and 9 with fire), 15 participants tried the condition with $50 \%$ of followers ( 7 without fire, and 8 with fire) and the last 14 participants tried the condition with $0 \%$ of followers (6 without fire, and 8 with fire).

\subsection{Questionnaire}

Besides collecting some basic personal information about the participants (e.g. age, gender, previous experience with computers, with virtual reality and playing video games), we were also interested in evaluating the overall realism of the virtual environment and the level of immersion experienced. Participants were asked their opinion regarding the realism of the virtual environment, the animations of the virtual characters, and the surrounding audio. Finally, we also wanted information about the feelings and reactions of the participants when the alarm went off. The list of question appears in table 1. All questions were rated on a scale of 0 (completely disagree) to 9 (completely agree). Questions 1 to 6 are about the perceived realism, embodiment and immersions. Questions 7 to 10 are about the participants behavior once the alarm goes off, in order to study human decision making based on the observed avatar behavior and the emergency situation.

Table 1: Questionnaire

\begin{tabular}{|l|l|}
\hline Q\# & Question/Statement \\
\hline Q1 & The overall the quality of the visualization was good. \\
\hline Q2 & $\begin{array}{l}\text { The quality of the VE makes it easy to perform the as- } \\
\text { signed tasks. }\end{array}$ \\
\hline Q3 & I consider the navigation in the VE to be intuitive. \\
\hline Q4 & At all times I felt in control of my avatar. \\
\hline Q5 & $\begin{array}{l}\text { The virtual humans' movement and appearance look re- } \\
\text { alistic. }\end{array}$ \\
\hline Q6 & $\begin{array}{l}\text { The surround sound helped me feel more immersed in the } \\
\text { VE. }\end{array}$ \\
\hline Q7 & $\begin{array}{l}\text { When the alarm went off I felt anxious/nervous as I did } \\
\text { not know what to do. }\end{array}$ \\
\hline Q8 & $\begin{array}{l}\text { The behavior of the other agents when the alarm went off } \\
\text { made me fell nervous. }\end{array}$ \\
\hline Q9 & $\begin{array}{l}\text { I felt the need to follow the other avatars instead of look- } \\
\text { ing for an exit by myself. }\end{array}$ \\
\hline
\end{tabular}




\subsection{Statistical Methods}

Two statistical tests have been performed to analyze the results obtained in our experiment. First, we checked whether the percentage of avatars running after the alarm went off has an impact on the participant's decision making. Since the input data can be divided into mutually exclusive and no overlapping categories, a Chi-Square test for association was performed. For the rest of analysis we performed ANOVA tests with 95\% confidence level and post-hoc Tukey analysis. We also applied a Bonferroni correction when needed.

\section{Results}

In this section we describe the results obtained from our study. Since we are studying decision making, each participant tried only one condition. This avoids our results being affected by what they could have learned in a previous experience. The conditions evaluated where a combination of percentage of avatars running towards exit F (0\%, $50 \%$ or $100 \%)$ with type of emergency (alarm with fire or without fire).

All participants were given the same questionnaire (see Table 1), followed by an open question where they could provide any comments or impressions about the experiment. Questions 1 to 6 allowed us to obtain information about the level of realism that participants perceived, as well as the level of immersion they experienced. Questions 7 to 9 where aimed to get information about the level of stress and decision making from the participants.

\subsection{Realism}

As the results show (Figure 9) most of the participants rated above 7 questions 1 to 6 , meaning they highly agreed that the environment, avatars movement (including their own) and surrounding sound made the experiment immersive and realistic.

Overall the participants commented that they found the environment very realistic and that the sound (announcements and train noise) made the experience very immersive. However they wished there had been some noise from conversation. They also commented that the avatar's behavior look realistic, but they found the animations a bit repetitive (there were walking animation, sitting down/standing up from benches, talking on the phone and a few idling). Some participants reported that in the scenario with no running avatars, they actually found it strange that people would not react to the alarm, which could have increased stress values. 


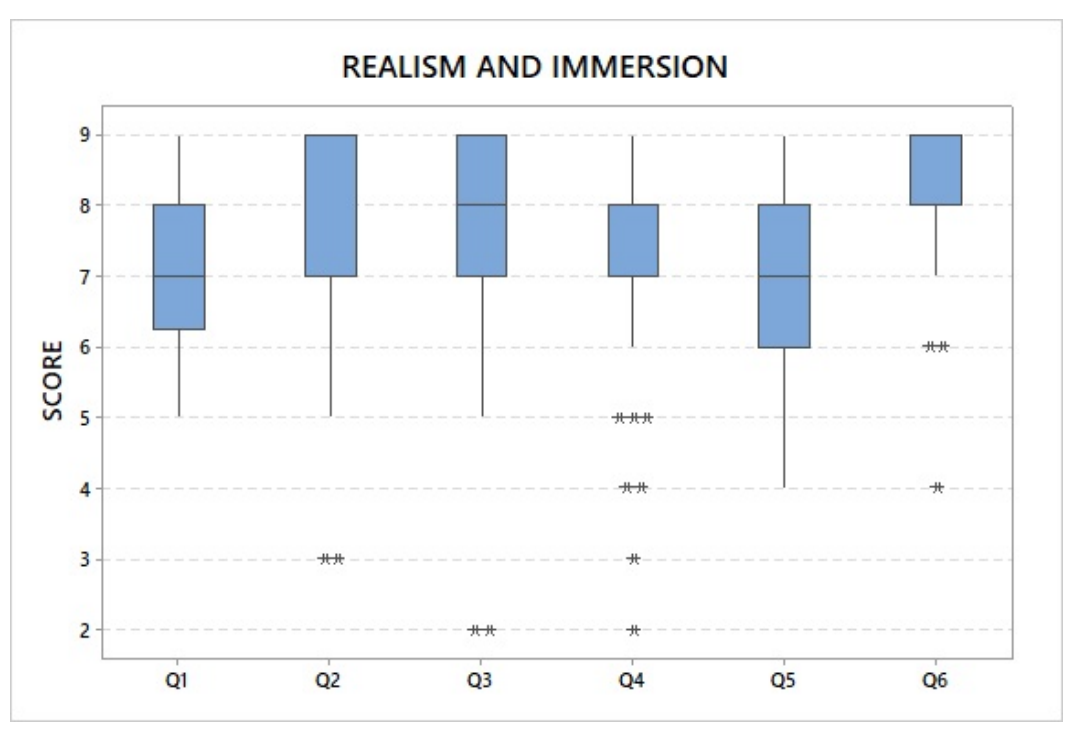

Fig. 9: Reported levels of realism and immersion.

\subsection{Decision making}

We analized how the amount of avatars running after the alarms goes off could have an impact on participants' decision making. Figure 10 shows a summary of our findings, with 'Check' meaning that the participant looked for the entrance doors before deciding whether to follow others or to search for an exit by themselves.

Our results showed that, when for the scenarios where $100 \%$ of the avatars ran towards exit $\mathrm{F}$ after the alarm goes off, $83.3 \%$ of the participants decided to follow them straight away, without even checking the main entrance or looking for a closer exit. This is consistent with the results obtained in [22]. Only one participant (that represents the 5.6\%) went first to the entrance doors and, after finding them locked, started looking for an exit. By the time the participant left the entrance area, all the virtual agents had already left the train station, therefore he did not have the chance to follow anybody. The rest of the participants $(11.2 \%$ found exit $\mathrm{A})$.

For the scenarios where $50 \%$ of the avatars ran, $53.3 \%$ of the participants checked first the main entrance and after finding it locked, they turned around and decided to follow the crowd. Therefore, by not having all avatars running, we observed the first attempts from participants to think by themselves. However, there were still 6 participants (that represented the $40 \%$ ) that did not even consider the possibility of leaving through the main entrance, and followed the running crowd immediately after the alarm went off. The rest of the participants $(6.67 \%)$ found exit A) 
Finally we tested the scenarios with $0 \%$ of followers, where all virtual agents simply continue with their errands without running for any exit after the alarm goes off. However, there is the possibility of having some avatars with their randomly assigned errand being to walk to exit $\mathrm{F}$ (but not running). Under this condition, $28.6 \%$ of participants started looking for an exit and found exit A. The other $64.3 \%$ of participants, first walked back to the main entrance doors and after finding them locked they started looking for an exit. Of that second group of participants, $67 \%$ eventually found exit A, and $33 \%$ went to exit $\mathrm{F}$ because they saw a few avatars walking towards that exit (this was verified from the comments they provided after the experiment). This shows that, even without a large crowd running, the fact that they appeared to be walking in a group was enough for those participants to decide to follow them (see Figure 10).

We performed a Chi-Square test of association to check whether the percentage of avatars running after the alarm goes off and the exit chosen by the participant are associated. The Pearson Chi-Square statistic is 8.08 and its corresponding p-value 0.018. The Likelihood-Ratio Chi-Square has a value of 8.17 and also has a p-value 0.017. We therefore can conclude that the percentage of avatars running affects the decision taken by the participants when choosing between looking for an exit (exit A) or following the crowd (exit F).

\section{Participants Evacuation Behavior}

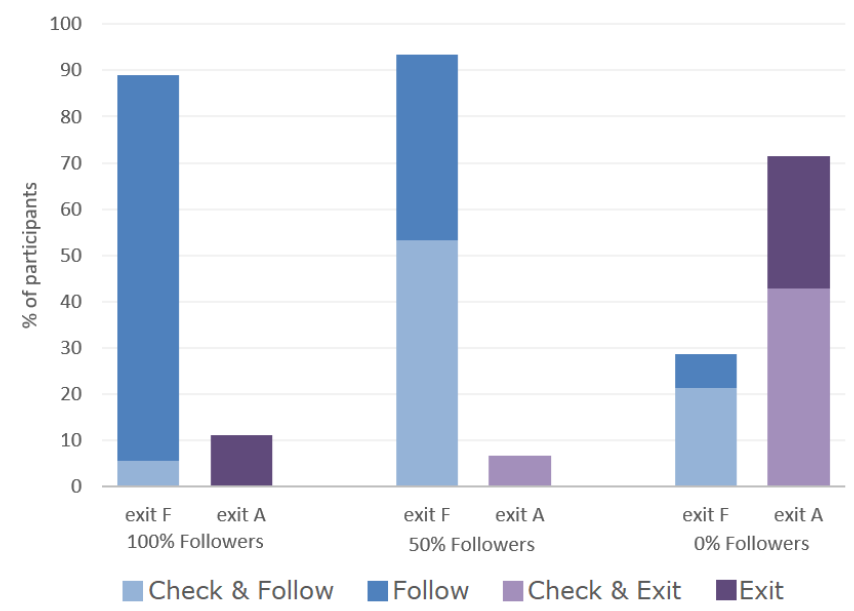

Fig. 10: Participants exit choice during the evacuation based on percentage of avatars running. 


\subsection{Level of nervousness}

We also analyzed the results of questions Q7 and Q8, in order to determine what aspects of the simulation had an impact on the level of nervousness experienced by the participants, and Q9 to study the relationship between egress behavior and nervousness (see Figure 11).

We first analyzed how the alarm going off affected nervousness and thus exit choice (Q7). We found that even though the average nervousness for participants that chose exit $\mathrm{F}$ was higher than for exit $\mathrm{A}\left(\mu_{F}=4.42, \mu_{A}=3.28\right)$, according to the one-way ANOVA this difference was not statistically significant, which means that there was not a clear influence on exit choice based on the alarm going off. For the case of reported nervousness after observing the avatars' behavior (Q8), the results showed that the average level of nervousness for those participants going to exit $\mathrm{F}$ was $\mu_{F}=4.06$ and for those going to exit A was $\mu_{A}=2.14$. In this case, the one-way ANOVA test and the Tukey post-hoc analysis resulted in $\rho=0.028$, and therefore this difference was statistically significant.

Regarding the impulse to follow others (Q9), the results of the one-way ANOVA showed that there is a statistically significant difference $(\rho<0.05)$ between the scores reported by the participants that decided to follow others to exit $\mathrm{F}\left(\mu_{F}=7.63\right)$ as opposed to those that looked for exit A by themselves $\left(\mu_{A}=0.57\right)$.

Exit choice vs. questions 7-9

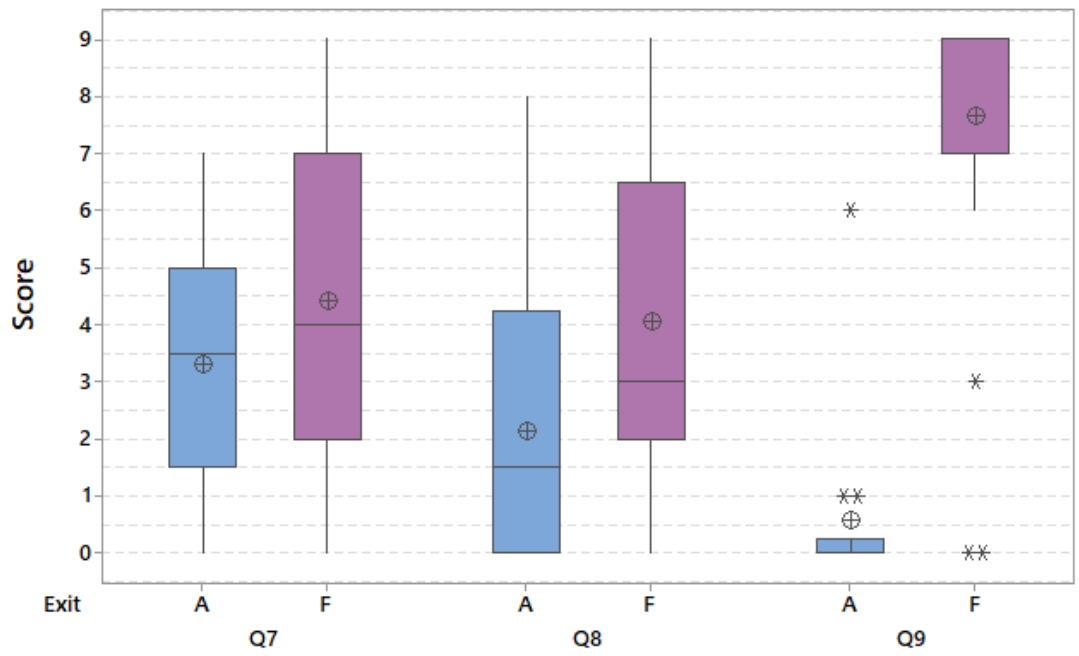

Fig. 11: Participant nervousness based on exit choice, due to the alarm (Q7), due to avatars' behavior (Q8). Q9 shows the impulse to follow others and its relationship to exit choice. The circle shows the mean values for each case. 
Figure 12 shows the results regarding nervousness levels based on avatars behavior (Q8), and impulse to follow avatars (Q9), both depending on the percentage of avatars running.

The one-way ANOVA performed for Q8 showed that this result is statistically significant with $\rho=0.01$. Meaning that as the percentage of avatars running increases, participants reported nervousness was higher. The average nervousness depending on the percentage of avatars running was: $\mu_{0}=$ $1.85, \mu_{50}=3.46, \mu_{100}=4.78$.

The one-way ANOVA performed for Q9, showed that the impulse to follow the avatars running was also higher as the percentage of avatars running increased (with $\rho=0.001$ ), with the average impulse to follow others being $\mu_{0}=2.43, \mu_{50}=6.2, \mu_{100}=7.4$. This is consistent with the comments we gathered from the participants, such as: "the avatars running gave the impression they knew where the exit was", or "in the real world I would have also followed people running, or even more an employee in uniform". Both for Q8 and Q9 the Bonferroni correction and the post-hoc analysis were applied.

Questions 8 and 9 vs. \% of avatars

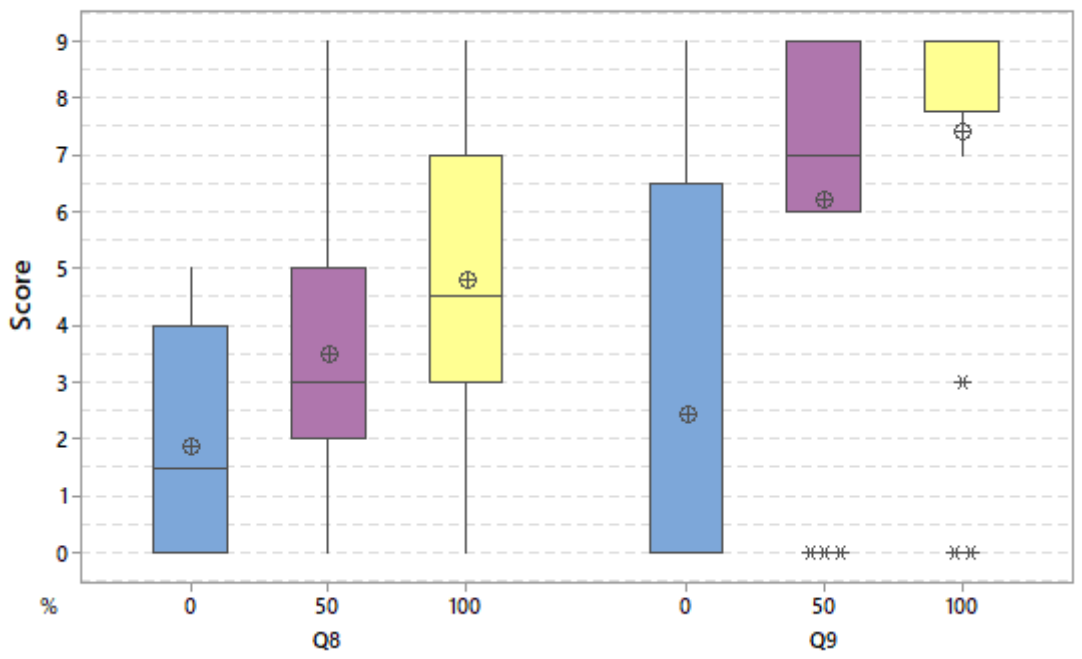

Fig. 12: Level of nervousness due to avatars' behavior (Q8) and impulse to follow avatars (Q9) based on the percentage of followers.

We then analyzed the level of nervousness of the participants when the alarm goes off, both with the presence of fire and without it. We performed a one-way ANOVA test to check whether the presence of fire would affect the level of nervousness of the participants. Surprisingly, we got $\rho>0.05$ showing that there is no statistically significant difference between stress levels with or without the presence of fire. Contrary to what we expected, the partici- 
pants did not get more nervous when they could clearly observe a danger. The graph in image 13 shows the difference in the level of nervousness between the participants of the two groups.

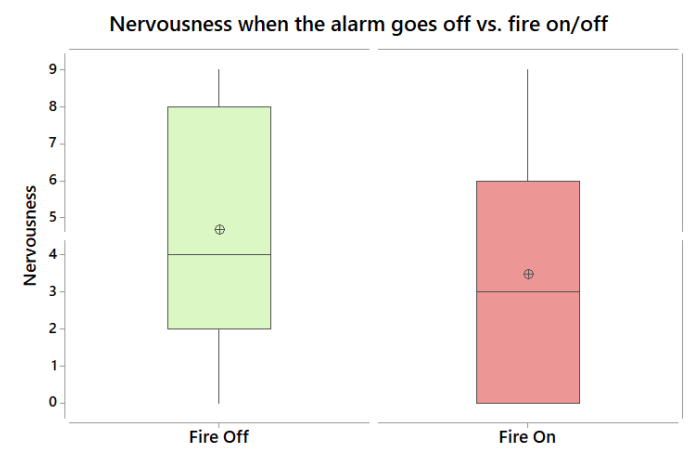

Fig. 13: Level of nervousness with fire on and fire off.

Studying the participants feedback and general comments that they provided after the experiment, we believe that the lack of difference in nervousness levels could be due to different causes:

1. Uncertainty may generate more anxiety. The first group of participants could not see what the danger was during the simulation. They could hear an alarm, see a flashing red light and some avatars running. But there were no cues regarding what the danger was or where it was located, thus they could not figure out what exactly was happening in the simulation which could be an important source of stress. On the contrary, the second group observed the fire on the train and saw the avatars fleeing from it. That provided enough information for the participants to be aware of the danger, and since it appears to be far away from their exit path, that may diminish stress. We believe that uncertainty can increase the level of stress in the participants and thus if the fire would have appeared as a stronger threat (e.g., in the main doors, or with plenty of smoke reducing visibility) the reported stress levels would have been higher.

2. Our participants were mostly gamers. $62.5 \%$ of the participants who performed the experiment answered with scores 8 and 9 to the question "Experience in games". This type of participants are used to play video games with many scenes of high tension so they did not get stressed by performing a simulation in which a few avatars run away and a harmless fire appears at one end of a train station. Graph in Fig. 14 shows that gamers presented a lower level of stress than non-gamers and a one-way ANOVA test and a Tukey post-hoc analysis showed that being a gamer affects the level of stress with $\rho<0.05$.

3. The level of realism on the avatars' behavior. Though the participants reported high levels of realism for the overall simulation, we consider that 
it is still relatively low to induce a high level of presence. For instance, as the alarm goes off, a fire breaks out on the train. However, the virtual avatars do not scream nor gesticulate, and there are no explosions from the fire. One participant commented that "I knew it was a game, so I didn't feel nervous" during the simulation. Another participant doing the $0 \%$ scenario reported "What I found awkward was the fact that when the alarm goes off and the fire starts, the avatars don't show any nervousness, they don't run away or anything similar". Avatars behavior was exactly the same in all simulations (with and without fire), this could be one more reason why there were not differences in stress level for the two scenarios. It is possible that stress is more linked to avatars' behavior than it is to the existence of fire.

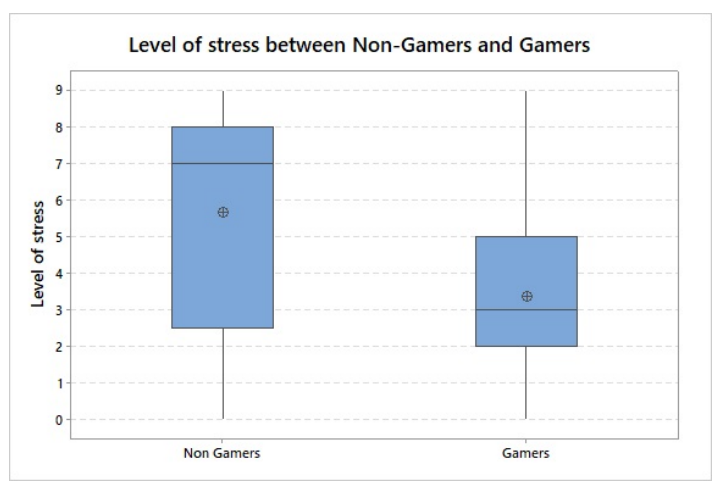

Fig. 14: Level of stress between non-gamers and gamers.

\section{Conclusions and Future Work}

In this paper we have carried out an experiment to study human decision making during an evacuation. We were interested to evaluate to what extend the behavior of a virtual crowd can affect individuals' decisions. Our results show how as the number of followers increases, there are more chances of people not taking their own decisions and simply follow what most of the people in a crowd are doing. It was also interesting to observe that the chances of exploring the environment are higher for people that experience low levels of stress in such situation. This observations should be further investigated, to obtain the relationship between personality and decision making strategies.

Another one of our observations was that, when participants are given a specific list of tasks to perform, they concentrate so much on it, that they do not spend enough time simply wandering around or inspecting their surroundings. This had as a consequence, that even though they all walked near an 
obvious exit, most participants did not notice it (the door was widely opened and they could see the buildings and street outside).

We have observed that the simple presence of fire, does not have an impact on the levels of stress experienced by participants. We believe that the reason was partly that the fire was far away and thus not seen as a threat. But more importantly, our results suggest that stress levels are highly linked to avatars behavior, and in our scenarios, avatars behavior was the same regardless of the presence or lack of fire. Presence research has proven that humans can behave in virtual reality in a similar manner as they would do in the real world. Our participants' reported high levels of presence in the environment. However, we believe that the lack of emotion and expressiveness from the avatars may have lessened the plausibility of the fire scenario. For example, in the real world, you would expect people to behave calm during an evacuation drill, but to show fear if there was a real fire emergency. As future work, we would like to test how having avatars showing panic (through screaming or facial expressions) could affect stress and thus decision making.

Our main conclusion from this work is that avatars' behavior can highly impact the participants response. So even though the overall realism is good for presence and immersion (render, sound, interaction, etc), special care should be put into the simulation and animation of avatars as it appears to be crucial if we want to develop plausible virtual environments for training and serious games.

\section{Acknowledgment}

This work was partly funded by the Spanish Ministry of Economy, Industry and Competitiveness under grant number TIN2017-88515-C2-1-R.

\section{References}

1. J. Ahn, N. Wang, D. Thalmann, and R. Boulic. Within-crowd immersive evaluation of collision avoidance behaviors. In Proceedings of the 11th ACM SIGGRAPH Int. Conference on Virtual-Reality Continuum and its Applications in Industry, pages 231238. ACM, 2012.

2. F. Argelaguet, A.-H. Olivier, G. Bruder, J. Pettré, and A. Lécuyer. Virtual proxemics: Locomotion in the presence of obstacles in large immersive projection environments. In $V R, 2015$.

3. J. Bruneau, A.-H. Olivier, and J. Pettre. Going through, going around: A study on individual avoidance of groups. IEEE transactions on visualization and computer graphics, 21(4):520-528, 2015.

4. V. J. Cassol, E. S. Testa, C. R. Jung, M. Usman, P. Faloutsos, G. Berseth, M. Kapadia, N. I. Badler, and S. R. Musse. Evaluating and optimizing evacuation plans for crowd egress. IEEE computer graphics and applications, 37(4):60-71, 2017.

5. R. B. Cialdini. Influence: The psychology of persuasion. 1993.

6. J. C. Coultas. When in rome... an evolutionary perspective on conformity. Group Processes \& Intergroup Relations, 7(4):317-331, 2004.

7. C. Ennis, C. Peters, and C. O'Sullivan. Perceptual evaluation of position and orientation context rules for pedestrian formations. In Proceedings of the 5th symposium on Applied perception in graphics and visualization, pages 75-82. ACM, 2008. 
8. C. Ennis, C. Peters, and C. O'Sullivan. Perceptual effects of scene context and viewpoint for virtual pedestrian crowds. ACM Transactions on Applied Perception (TAP), 8(2):10:1-10:22, 2011.

9. M. Gonzalez-Franco and J. Lanier. Model of illusions and virtual reality. Frontiers in Psychology, 8:1125, 2017.

10. M. Gonzalez-Franco, M. Slater, M. E. Birney, D. Swapp, S. A. Haslam, and S. D. Reicher. Participant concerns for the learner in a virtual reality replication of the milgram obedience study. PloS one, 13(12):e0209704, 2018.

11. N. Gupta, A. Singh, and S. Butail. The effect of instructional priming on postural responses to virtual crowds. In Virtual Humans and Crowds for Immersive Environments (VHCIE), pages 1-8. IEEE, 2017.

12. S. J. Guy, J. Chhugani, S. Curtis, P. Dubey, M. Lin, and D. Manocha. Pledestrians: a least-effort approach to crowd simulation. In Proceedings of the 2010 ACM SIGGRAPH/Eurographics symposium on computer animation, pages 119-128. Eurographics Association, 2010.

13. S. J. Guy, S. Kim, M. C. Lin, and D. Manocha. Simulating heterogeneous crowd behaviors using personality trait theory. In Proceedings of the 2011 ACM SIGGRAPH/Eurographics symposium on computer animation, pages 43-52. ACM, 2011.

14. S. J. Guy, J. Van Den Berg, W. Liu, R. Lau, M. C. Lin, and D. Manocha. A statistical similarity measure for aggregate crowd dynamics. ACM Transactions on Graphics (TOG), 31(6):190, 2012.

15. B. Haworth, M. Usman, G. Berseth, M. Kapadia, and P. Faloutsos. Evaluating and optimizing level of service for crowd evacuations. In Proceedings of the 8th ACM SIGGRAPH Conference on Motion in Games, MIG '15, pages 91-96, New York, NY, USA, 2015. ACM.

16. A. E. Kazdin and A. E. Kazdin. Encyclopedia of psychology, volume 2. American Psychological Association Washington, DC, 2000.

17. P. Knob, M. Balotin, and S. R. Musse. Simulating crowds with ocean personality traits. In Proceedings of the 18th International Conference on Intelligent Virtual Agents, pages 233-238. ACM, 2018.

18. M. Kyriakou and Y. Chrysanthou. How responsiveness, group membership and gender affect the feeling of presence in immersive virtual environments populated with virtual crowds. In Proceedings of the 11th Annual International Conference on Motion, Interaction, and Games, page 12. ACM, 2018.

19. A. Lerner, Y. Chrysanthou, A. Shamir, and D. Cohen-Or. Context-dependent crowd evaluation. In Computer Graphics Forum, pages 2197-2206. Wiley Online Library, 2010.

20. J. Llobera, B. Spanlang, G. Ruffini, and M. Slater. Proxemics with multiple dynamic characters in an immersive virtual environment. ACM Transactions on Applied Perception (TAP), 8(1):3, 2010.

21. J. L. Maples-Keller, C. W. Yasinski, N. Manjin, and B. O. Rothbaum. Virtual realityenhanced extinction of phobias and post-traumatic stress. Neurotherapeutics, 14:554$563,2017$.

22. M. Moussaïd, M. Kapadia, T. Thrash, R. W. Sumner, M. Gross, D. Helbing, and C. Hölscher. Crowd behaviour during high-stress evacuations in an immersive virtual environment. Journal of The Royal Society Interface, 13(122):20160414, 2016.

23. S. Narang, A. Best, T. Randhavane, A. Shapiro, and D. Manocha. Pedvr: Simulating gaze-based interactions between a real user and virtual crowds. In Proceedings of the 22nd ACM conference on virtual reality software and technology, pages 91-100. ACM, 2016.

24. A.-H. Olivier, J. Bruneau, G. Cirio, and J. Pettré. A virtual reality platform to study crowd behaviors. Transportation Research Procedia, 2:114-122, 2014.

25. A.-H. Olivier, A. Marin, A. Crétual, A. Berthoz, and J. Pettré. Collision avoidance between two walkers: Role-dependent strategies. Gait and Posture, 38(4):751 - 756, 2013.

26. J. Ondřej, J. Pettré, A.-H. Olivier, and S. Donikian. A synthetic-vision based steering approach for crowd simulation. ACM Transactions on Graphics (TOG), 29(4):123, 2010 . 
27. N. Pelechano and J. M. Allbecky. Feeling crowded yet?: crowd simulations for vr. In Virtual Humans and Crowds for Immersive Environments (VHCIE), pages 17-21. IEEE, 2016.

28. N. Pelechano and N. I. Badler. Modeling crowd and trained leader behavior during building evacuation. IEEE computer graphics and applications, 26(6), 2006.

29. N. Pelechano, C. Stocker, J. Allbeck, and N. Badler. Being a part of the crowd: towards validating vr crowds using presence. In Proceedings of the 7th international joint conference on Autonomous agents and multiagent systems-Volume 1, pages 136-142, 2008.

30. N. Pelechano Gómez, C. Stocker, J. Allbeck, and N. Badler. Feeling crowded? exploring presence in virtual crowds. In Proceedings of PRESENCE 2007, pages 373-376, 2007.

31. A. Ríos, M. Palomar, and N. Pelechano. Users' locomotor behavior in collaborative virtual reality. In Proceedings of the 11th Annual International Conference on Motion, Interaction, and Games, page 15. ACM, 2018.

32. F. A. Rojas and H. S. Yang. Immersive human-in-the-loop hmd evaluation of dynamic group behavior in a pedestrian crowd simulation that uses group agent-based steering. In Proceedings of the 12th ACM SIGGRAPH International Conference on Virtual-Reality Continuum and Its Applications in Industry, VRCAI '13, pages 31-40, New York, NY, USA, 2013. ACM.

33. A. Ríos and N. Pelechano. Follower behavior in a virtual environment. In Virtual Humans and Crowds in Immersive Environments (VHCIE), March 2018.

34. N. Sohre, C. Mackin, V. Interrante, and S. J. Guy. Evaluating collision avoidance effects on discomfort in virtual environments. In Virtual Humans and Crowds for Immersive Environments (VHCIE), pages 1-5. IEEE, 2017.

35. A. Steed, Y. Pan, Z. Watson, and M. Slater. "we wait" - the impact of character responsiveness and self embodiment on presence and interest in an immersive news experience. Frontiers in Robotics and AI, 5:112, 2018.

36. A. Turner and A. Penn. Encoding natural movement as an agent-based system: an investigation into human pedestrian behaviour in the built environment. Environment and planning B: Planning and Design, 29(4):473-490, 2002.

37. A. Turner and A. Penn. Evolving direct perception models of human behavior in building systems. In Pedestrian and Evacuation Dynamics 2005, pages 411-422. Springer, 2007.

38. W. G. Van Toll, A. F. Cook, and R. Geraerts. Real-time density-based crowd simulation. Computer Animation and Virtual Worlds, 23(1):59-69, 2012.

39. D. Wolinski, S. J Guy, A.-H. Olivier, M. Lin, D. Manocha, and J. Pettré. Parameter estimation and comparative evaluation of crowd simulations. In Computer Graphics Forum, pages 303-312. Wiley Online Library, 2014. 